\title{
PRODUCT TWISTOR SPACES AND WEYL GEOMETRY
}

\author{
JOHANN DAVIDOV
}

\begin{abstract}
Motivated by generalized geometry (à la Hitchin), we discuss the integrability conditions for four natural almost complex structures on the product bundle $\mathcal{Z} \times \mathcal{Z} \rightarrow M$, where $\mathcal{Z}$ is the twistor space of a Riemannian 4manifold $M$ endowed with a metric connection $D$ with skew-symmetric torsion. These structures are defined by means of the connection $D$ and four (Kähler) complex structures on the fibres of this bundle. Their integrability conditions are interpreted in terms of Weyl geometry and this is used to supply examples satisfying the conditions.
\end{abstract}

2010 Mathematics Subject Classification 53C28; 53C15, 53D18.

Key words: twistor spaces, almost complex structures, skew-symmetric torsion, Weyl geometry.

Dedicated to the memory of Thomas Friedrich

\section{INTRODUCTION}

The motivation for this paper comes from generalized geometry in the sense of N. Hitchin [16].

Recall that a generalized metric [15, 31] on a smooth manifold $M$ is a subbundle $E$ of $T M \oplus T^{*} M$ such that the restriction to $E$ of the metric $\langle X+\alpha, Y+\beta\rangle=$ $\alpha(Y)+\beta(X)$ is positive definite, $X, Y \in T M, \alpha, \beta \in T^{*} M$. Every generalized metric is determined by a positive definite metric $g$ and a skew-symmetric 2 -form $\Theta$ on $M$ such that $E=\left\{X+\imath_{X} g+\imath_{X} \Theta: X \in T M\right\}$. Moreover, $E$ determines a connection on $M$ which is metric with respect to $g$ and whose torsion form is skew-symmetric, equal to $d \Theta$, see [17].

Given a generalized metric $E$, denote by $\mathcal{G}(E)$ the bundle over $M$ whose fibre at a point $p \in M$ consists of generalized complex structures $J$ on the tangent space $T_{p} M$ compatible with the generalized metric $E_{p}$, the fibre of $E$ at $p$, in the sense that $J E_{p} \subset E_{p}$. The bundle $\mathcal{G}(E)$, called the generalized twistor space of $(M, E)$ in $[8$, admits a generalized almost complex structure $\mathcal{J}$ defined by means of the connection determined by $E$. This structure resembles the Atiyah-HitchinSinger almost complex structure on the usual twistor space [1]. The integrability condition for $\mathcal{J}$ (in the sense of generalized geometry) has been found in [9] in the case when $\operatorname{dim} M=4$ and $\Theta=0$, and in $[8]$ in the general case. Besides the generalized almost complex structure $\mathcal{J}$, the manifold $\mathcal{G}(E)$ admits four natural almost complex structure $\mathfrak{J}^{m}$ in the usual sense since $\mathcal{G}(E)$ is diffeomorphic to the product bundle $\mathcal{Z} \times \mathcal{Z} \rightarrow M$ where $\mathcal{Z}$ is the (usual) twistor space of the Riemannian manifold $(M, g)$, see, for example, [8]. They are defined by means of a metric

The author is partially supported by the National Science Fund, Ministry of Education and Science of Bulgaria under contract DN 12/2. 
connection $D$ on $M$ with skew-symmetric torsion and four complex structures (in fact, Kähler ones) on the fibres of $\mathcal{Z} \times \mathcal{Z}$, and are not products of almost complex structures on $\mathcal{Z}$. Two of these structures, denoted by $\mathcal{J}^{1}$ and $\mathcal{J}^{2}$, can be considered as analogs of the Atiyah-Hitchin-Singer almost complex structure, while $\mathcal{J}^{3}$ and $\mathcal{J}^{4}$ correspond to the Eells-Salamon almost complex structure on $\mathcal{Z}$ [11. In the this paper, we deal with the classical integrability problem for these structures. The integrability condition for the almost complex structure $J^{1}$ has been found in 9] when $\operatorname{dim} M=4$ and $\Theta=0$ (so $D$ is the Levi-Civita connection of $g$ ). In the present paper, we also consider the most interesting case of an oriented four-dimensional manifold, but the connection used here has skew-symmetric torsion. In order to find the corresponding integrability condition, we briefly discuss the Atiyah-HitchinSinger almost complex structure on $\mathcal{Z}$ defined by means of a metric connection with skew-symmetric torsion. The integrability condition for this structure does not depend on the torsion and is (anti-) self-duality of the metric. This, suggested by certain facts, formally follows easily from a result in 10. It is a simple observation that the almost complex structures $\mathcal{J}^{3}$ and $\mathcal{J}^{4}$ are never integrable. On the other hand, the integrability conditions for the restrictions of $\mathcal{J}^{1}$ and $\mathcal{J}^{2}$ to the connected components of $\mathcal{Z} \times \mathcal{Z}$ involve (anti-) self-duality of the metric and certain relations between the Ricci curvature of the Levi-Civita connection and the torsion of the given connection. The latter are interpreted in terms of Weyl geometry and this is used to supply examples of manifolds satisfying the integrability conditions.

\section{Preliminaries}

Let $(M, g)$ be an oriented Riemannian manifold of dimension four. The metric $g$ induces a metric on the bundle of two-vectors $\pi: \Lambda^{2} T M \rightarrow M$ by the formula

$$
g\left(v_{1} \wedge v_{2}, v_{3} \wedge v_{4}\right)=\frac{1}{2} \operatorname{det}\left[g\left(v_{i}, v_{j}\right)\right],
$$

the factor $1 / 2$ being chosen in consistence with [6].

Let $*: \Lambda^{k} T M \rightarrow \Lambda^{4-k} M, k=0, \ldots, 4$, be the Hodge star operator. Its restriction to $\Lambda^{2} T M$ is an involution, thus we have the orthogonal decomposition

$$
\Lambda^{2} T M=\Lambda_{-}^{2} T M \oplus \Lambda_{+}^{2} T M,
$$

where $\Lambda_{ \pm}^{2} T M$ are the subbundles of $\Lambda^{2} T M$ corresponding to the ( \pm 1$)$-eigenvalues of the operator *

Let $\left(E_{1}, E_{2}, E_{3}, E_{4}\right)$ be a local oriented orthonormal frame of $T M$. Set

(1) $s_{1}^{ \pm}=E_{1} \wedge E_{2} \pm E_{3} \wedge E_{4}, \quad s_{2}^{ \pm}=E_{1} \wedge E_{3} \pm E_{4} \wedge E_{2}, \quad s_{3}^{ \pm}=E_{1} \wedge E_{4} \pm E_{2} \wedge E_{3}$.

Then $\left(s_{1}^{ \pm}, s_{2}^{ \pm}, s_{3}^{ \pm}\right)$is a local orthonormal frame of $\Lambda_{ \pm}^{2} T M$. This frame defines an orientation on $\Lambda_{ \pm}^{2} T M$ which does not depend on the choice of the frame $\left(E_{1}, E_{2}, E_{3}, E_{4}\right)$ (see, for example, [7). We call this orientation "canonical".

For every $a \in \Lambda^{2} T M$, define a skew-symmetric endomorphism of $T_{\pi(a)} M$ by

$$
g\left(K_{a} X, Y\right)=2 g(a, X \wedge Y), \quad X, Y \in T_{\pi(a)} M .
$$

Denoting by $G$ the standard metric $-\frac{1}{2}$ Trace $P Q$ on the space of skew-symmetric endomorphisms, we have $G\left(K_{a}, K_{b}\right)=2 g(a, b)$ for $a, b \in \Lambda^{2} T M$. If $a \in \Lambda^{2} T M$ is of unit length, then $K_{a}$ is a complex structure on the vector space $T_{\pi(a)} M$ compatible with the metric $g$, i.e., $g$-orthogonal. Conversely, the 2 -vector $a$ dual to 
one half of the fundamental 2-form of such a complex structure is a unit vector in $\Lambda^{2} T M$. Therefore the unit sphere bundle $\mathcal{Z}$ of $\Lambda^{2} T M$ parametrizes the complex structures on the tangent spaces of $M$ compatible with the metric $g$. This bundle is called the twistor space of the Riemannian manifold $(M, g)$. Since $M$ is oriented, the manifold $\mathcal{Z}$ has two connected components $\mathcal{Z}_{ \pm}$called the positive and the negative twistor spaces of $(M, g)$. These are the unit sphere subbundles of $\Lambda_{ \pm}^{2} T M$. The bundle $\mathcal{Z}_{ \pm} \rightarrow M$ parametrizes the complex structures on the tangent spaces of $M$ compatible with the metric and \pm the orientation via the correspondence $\mathcal{Z}_{ \pm} \ni \sigma \rightarrow K_{\sigma}$

The vertical space $\mathcal{V}_{\sigma}=\left\{V \in T_{\sigma} \mathcal{Z}_{ \pm}: \pi_{*} V=0\right\}$ of the bundle $\pi: \mathcal{Z}_{ \pm} \rightarrow M$ at a point $\sigma$ is the tangent space to the fibre of $\mathcal{Z}_{ \pm}$through $\sigma$. Thus, considering $T_{\sigma} \mathcal{Z}_{ \pm}$ as a subspace of $T_{\sigma}\left(\Lambda_{ \pm}^{2} T M\right), \mathcal{V}_{\sigma}$ is the orthogonal complement of $\mathbb{R} \sigma$ in $\Lambda_{ \pm}^{2} T_{\pi(\sigma)} M$.

Let $D$ be a metric connection on $(M, g)$. The induced connection on $\Lambda^{2} T M$ will also be denoted by $D$. If $\left(s_{1}^{ \pm}, s_{2}^{ \pm}, s_{3}^{ \pm}\right)$is the orthonormall frame of $\Lambda_{ \pm}^{2} T M$ defined by means of an oriented orthonormal frame $\left(E_{1}, \ldots, E_{4}\right)$ of $T M$ via (1), we have $g\left(D_{X} s_{i}^{+}, s_{j}^{-}\right)=g\left(D_{X} s_{i}^{-}, s_{j}^{+}\right)=0$ and $g\left(D_{X} s_{i}^{ \pm}, s_{j}^{ \pm}\right)=-g\left(D_{X} s_{j}^{ \pm}, s_{i}^{ \pm}\right)$for every $X \in T M$ and every $i, j=1,2,3$. Therefore the connection $D$ preserves the bundles $\Lambda_{ \pm}^{2} T M$ and induces a metric connection on each of these bundles denoted again by $D$. Let $\sigma \in \mathcal{Z}_{ \pm}$, and let $s$ be a local section of $\mathcal{Z}_{ \pm}$such that $s(p)=\sigma$ where $p=\pi(\sigma)$. Considering $s$ as a section of $\Lambda_{ \pm}^{2} T M$, we have $D_{X} s \perp s(p)$, i.e., $D_{X} s \in \mathcal{V}_{\sigma}$ for every $X \in T_{p} M$ since $s$ has a constant length. Moreover, $X_{\sigma}^{h}=s_{*} X-D_{X} s \in T_{\sigma} \mathcal{Z}_{ \pm}$is the horizontal lift of $X$ at $\sigma$ with respect the connection $D$ on $\Lambda_{ \pm}^{2} T M$. Thus, the horizontal distribution of $\Lambda_{ \pm}^{2} T M$ with respect to $D$ is tangent to the twistor space $\mathcal{Z}_{ \pm}$. In this way, we have the decomposition $T \mathcal{Z}_{ \pm}=\mathcal{H} \oplus \mathcal{V}$ of the tangent bundle of $\mathcal{Z}_{ \pm}$into horizontal and vertical components. The horizontal and vertical parts of a tangent vector $A \in T \mathcal{Z}_{ \pm}$will be denoted by $\mathcal{H} A$ and $\mathcal{V} A$, respectively.

For $\sigma \in \mathcal{Z}_{ \pm}$, the horizontal space $\mathcal{H}_{\sigma}$ is isomorphic to the tangent space $T_{\pi(\sigma)} M$ via the differential $\pi_{* \sigma}$. The vertical space $\mathcal{V}_{\sigma}$ is tangent to the unit sphere in the 3-dimensional vector space $\left(\Lambda_{ \pm}^{2} T_{\pi(\sigma)} M, g\right)$, and we denote by $\mathcal{J}_{\sigma}$ the standard complex structure of the unit sphere restricted to $\mathcal{V}_{\sigma}$. It is given by

$$
\mathcal{J}_{\sigma} V= \pm(\sigma \times V), \quad V \in \mathcal{V}_{\sigma},
$$

where $\times$ is the usual vector-cross product on the 3 -dimensional Euclidean space $\left(\Lambda_{ \pm}^{2} T_{\pi(\sigma)} M, g\right)$ endowed with its canonical orientation.

Convention. In what follow, we shall freely identify the bundle $\Lambda^{2} T M$ with the bundle $A(T M)$ of $g$-skew-symmetric endomorphism of $T M$ by means of the isomorphism $a \rightarrow K_{a}$ defined by (2).

Using the basis (11), it is easy to check that if $a, b \in \Lambda_{ \pm}^{2} T_{p} M$, the isomorphism $\Lambda^{2} T M \cong A(T M)$ sends $a \times b$ to $\pm \frac{1}{2}\left[K_{a}, K_{b}\right]$. In the case when $a \in \Lambda_{+}^{2} T_{p} M$, $b \in \Lambda_{-}^{2} T_{p} M$, the endomorphisms $K_{a}$ and $K_{b}$ of $T_{p} M$ commute. If $a, b \in \Lambda_{ \pm} T_{p} M$,

$$
K_{a} \circ K_{b}=-g(a, b) I d \pm K_{a \times b} .
$$

In particular, $K_{a}$ and $K_{b}, a, b \in \Lambda_{ \pm} T_{p} M$, anti-commute if and only if $a$ and $b$ are orthogonal.

The 6-manifold $\mathcal{Z}_{ \pm}$admits two almost complex structures $\mathcal{J}_{1}$ and $\mathcal{J}_{2}$ introduced, respectively, by Atiyah-Hitchin-Singer [1, and Eells-Salamon [11] in the case when the connection $D$ is the Levi-Civita connection of $(M, g)$. On a horizontal space $\mathcal{H}_{\sigma}, \sigma \in \mathcal{Z}_{ \pm}$, the structures $\mathcal{J}_{1}$ and $\mathcal{J}_{2}$ are both defined as the lift to $\mathcal{H}_{\sigma}$ of the 
complex structure $K_{\sigma}$ on $T_{\pi(\sigma)} M$. On a vertical space $\mathcal{V}_{\sigma}, \mathcal{J}_{1}$ is defined to be the complex structure $\mathcal{J}_{\sigma}$ of the fibre through $\sigma$, while $\mathcal{J}_{2}$ is defined as the conjugate complex structure, i.e., $\mathcal{J}_{2} \mid \mathcal{V}_{\sigma}=-\mathcal{J}_{\sigma}$. Thus, for $J \in \mathcal{Z}_{ \pm}$,

$$
\begin{gathered}
\mathcal{J}_{n}\left|\mathcal{H}_{J}=\left(\pi_{*} \mid \mathcal{H}_{J}\right)^{-1} \circ J \circ \pi_{*}\right| \mathcal{H}_{J} \\
\mathcal{J}_{n} V=(-1)^{n+1}(J \circ V) \text { for } V \in \mathcal{V}_{\mathcal{J}}, \quad n=1,2 .
\end{gathered}
$$

Suppose that $D$ is the Levi-Civita connection $\nabla$ of $(M, g)$. By a result of EellsSalamon [11, the almost complex structure $\mathcal{J}_{2}$ is never integrable, so it does not come from a complex structure. Nevertheless, $\mathcal{J}_{2}$ is very useful for constructing harmonic maps. The integrability condition for $\mathcal{J}_{1}$ has been found by AtiyahHitchin-Singer [1. To state their result, we first recall the well-known curvature decomposition in dimension four. For the curvature tensor $R^{D}$ of a connection $D$ on $M$, we adopt the following definition: $R^{D}(X, Y)=D_{[X, Y]}-\left[D_{X}, D_{Y}\right]$. The curvature operator $\mathcal{R}^{D}$ corresponding to the curvature tensor is the endomorphism of $\Lambda^{2} T M$ defined by

$$
g\left(\mathcal{R}^{D}(X \wedge Y), Z \wedge U\right)=g\left(R^{D}(X, Y) Z, U\right), \quad X, Y, Z, U \in T M .
$$

Now, let $\nabla$ be the Levi-Civita connection of $(M, g)$. Denote by $\rho: T M \rightarrow T M$ its Ricci operator, $g(\rho(X), Y)=\operatorname{Ricci}(X, Y)$, and by $s$ the scalar curvature. Then the endomorphism $\mathcal{B}: \Lambda^{2} T M \rightarrow \Lambda^{2} T M$ corresponding to the traceless Ricci tensor is given by

$$
\mathcal{B}(X \wedge Y)=\rho(X) \wedge Y+X \wedge \rho(Y)-\frac{s}{2} X \wedge Y .
$$

Note that $\mathcal{B}$ sends $\Lambda_{ \pm}^{2} T M$ into $\Lambda_{\mp}^{2} T M$. Let $\mathcal{W}: \Lambda^{2} T M \rightarrow \Lambda^{2} T M$ be the endomorphism corresponding to the Weyl conformal tensor. Denote the restriction of $\mathcal{W}$ to $\Lambda_{ \pm}^{2} T M$ by $\mathcal{W}_{ \pm}$, so $\mathcal{W}_{ \pm}$sends $\Lambda_{ \pm}^{2} T M$ to $\Lambda_{ \pm}^{2} T M$ and vanishes on $\Lambda_{\mp}^{2} T M$

It is well known that the curvature operator decomposes as (28], see, e.g., [3, Chapter $1 \mathrm{H}]$ )

$$
\mathcal{R}^{\nabla}=\frac{s}{6} I d+\mathcal{B}+\mathcal{W}_{+}+\mathcal{W}_{-}
$$

Note that this differs from [3] by a factor $1 / 2$ because of the factor $1 / 2$ in our definition of the induced metric on $\Lambda^{2} T M$.

The Riemannian manifold $(M, g)$ is Einstein exactly when $\mathcal{B}=0$. It is called selfdual (anti-self-dual) if $\mathcal{W}_{-}=0$ (resp. $\mathcal{W}_{+}=0$ ). The self-duality (anti-self-duality) condition is invariant under conformal changes of the metric since the Weyl tensor is so.

Note that changing the orientation of $M$ interchanges the roles of $\Lambda_{-}^{2} T M$ and $\Lambda_{+}^{2} T M$ (respectively, of $\mathcal{Z}_{-}$and $\mathcal{Z}_{+}$), hence the roles of $\mathcal{W}_{-}$and $\mathcal{W}_{+}$.

The famous Atiyah-Hitchin-Singer theorem [1] states that the almost complex structure $\mathcal{J}_{1}$ on $\mathcal{Z}_{-}$(resp. $\mathcal{Z}_{+}$) is integrable if and only if $(M, g)$ is self-dual (resp. anti-self-dual).

\section{Technical Lemmas}

Let $D$ be a metric connection on $(M, g)$, and consider the almost complex structures $\mathcal{J}_{n}, n=1,2$, defined on the positive twistor space $\mathcal{Z}_{+}$by means of the 
connection $D$. Denote by $\mathcal{N}_{n}$ the Nijenhuis tensor of the almost complex structure $\mathcal{J}_{n}$ :

$$
\mathcal{N}_{n}(A, B)=-[A, B]+\left[\mathcal{J}_{n} A, \mathcal{J}_{n} B\right]-\mathcal{J}_{n}\left[\mathcal{J}_{n} A, B\right]-\mathcal{J}_{n}\left[A, \mathcal{J}_{n} B\right] .
$$

Let $T$ be the torsion tensor of the connection $D$.

Lemma 1. (2, 6, 10, 13, 24]) Let $J \in \mathcal{Z}_{+}, V, W \in \mathcal{V}_{J}$, and let $X, Y \in T_{\pi(J)} M$. Then

(i) $\mathcal{H} \mathcal{N}_{n}\left(X^{h}, Y^{h}\right)_{J}=(T(X, Y)-T(J X, J Y)+J T(J X, Y)+J T(X, J Y))_{J}^{h}$.

(ii) $\mathcal{V N}_{n}\left(X^{h}, Y^{h}\right)_{J}=-R^{D}(X, Y) J+R^{D}(J X, J Y) J$

$$
-\mathcal{J}_{n} R^{D}(J X, Y) J-\mathcal{J}_{n} R^{D}(X, J Y) J .
$$

(iii) $\mathcal{N}_{n}\left(X_{J}^{h}, V\right)=\left[(-1)^{n}+1\right](J V X)_{J}^{h}$.

(iv) $\mathcal{N}_{n}(V, W)=0$.

Corollary 1. The almost complex structure $\mathcal{J}_{2}$ is never integrable.

Proof. If $\left(E_{1}, \ldots, E_{4}\right)$ is an oriented orthonormal frame, set $J=E_{1} \wedge E_{2}+E_{3} \wedge E_{4}$, $V=E_{1} \wedge E_{3}+E_{4} \wedge E_{2}$. Then $\mathcal{V N}_{2}\left(E_{1}^{h}, V\right)_{J}=2\left(E_{4}\right)_{J}^{h} \neq 0$.

\section{Integrability of the Atiyah-Hitchin-Singer almost COMPleX} STRUCTURE ON THE TWISTOR SPACES OF A RIEMANNIAN 4-MANIFOLD WITH SKEW-SYMMETRIC TORSION

Suppose that the metric connection $D$ has (totally) skew-symmetric torsion, i.e., the trilinear form

$$
\mathcal{T}(X, Y, Z)=g(T(X, Y), Z), \quad X, Y, Z \in T M,
$$

is skew-symmetric. Let $\nabla$ be the Levi-Civita connection of $(M, g)$. Then

$$
D_{X} Y=\nabla_{X} Y+\frac{1}{2} T(X, Y) \text {. }
$$

The difference between the connections $D$ and $\nabla$ satisfies the following identity.

Lemma 2. If the torsion $T$ is skew-symmetric, then for every $J \in \mathcal{Z}_{+}$and every $X, Y \in T_{\pi(J)} M$,

$$
T(X, Y)-T(J X, J Y)+J T(J X, Y)+J T(X, J Y)=0
$$

Proof. Take an oriented orthonormal basis $\left(E_{1}, \ldots, E_{4}\right)$ of $T_{\pi(J)} M$ such that $E_{2}=$ $J E_{1}, E_{4}=J E_{3}$. Then it is easy to check that

$$
\mathcal{T}\left(E_{i}, E_{j}, E_{k}\right)-\mathcal{T}\left(J E_{i}, J E_{j}, E_{k}\right)-\mathcal{T}\left(J E_{i}, E_{j}, J E_{k}\right)-\mathcal{T}\left(E_{i}, J E_{j}, J E_{k}\right)=0
$$

for every $i, j, k=1, \ldots, 4$.

Now, it follows from [10, Lemma 2.4] that the connections $D$ and $\nabla$ define the same almost complex structure on the twistor space. Thus, by the Atiyah-HitchinSinger theorem [1], we have:

Theorem 1. Let $(M, g)$ be an oriented Riemannian 4-manifold endowed with a metric connection with skew-symmetric torsion. The Atiyah-Hitchin-Singer almost complex structure $\mathcal{J}_{1}$ on the twistor space $\mathcal{Z}_{+}$is integrable if and only if the metric $g$ is anti-self-dual. 
Remark. This result can also be proved by means of Lemmas 1 and 2 , and the following relation between the curvature tensors $R^{D}$ and $R^{\nabla}$ of the connections $D$ and $\nabla$ :

$$
\begin{gathered}
g\left(R^{D}(X, Y) Z, U\right)=g\left(R^{\nabla}(X, Y) Z, U\right) \\
-\frac{1}{2}\left[\left(\nabla_{X} \mathcal{T}\right)(Y, Z, U)-\left(\nabla_{Y} \mathcal{T}\right)(X, Z, U)\right] \\
+\frac{1}{4} \sum_{i=1}^{4}\left[\mathcal{T}\left(X, U, E_{i}\right) \mathcal{T}\left(Y, Z, E_{i}\right)-\mathcal{T}\left(X, Z, E_{i}\right) \mathcal{T}\left(Y, U, E_{i}\right)\right],
\end{gathered}
$$

where $X, Y, Z, U \in T_{p} M$ and $\left\{E_{1}, \ldots, E_{4}\right\}$ is an orthonormal basis of $T_{p} M$.

\section{INTEgRABILITy of NATURAL ALMOST COMPLEX STRUCTURES ON A PRODUCT TWISTOR SPACE}

Every metric connection $D$ on $(M, g)$ induces a metric connection (again denoted by $D$ ) on the product bundle $\pi: \Lambda^{2} T M \times \Lambda^{2} T M \rightarrow M$, which preserves the four subbundles $\Lambda_{ \pm}^{2} T M \times \Lambda_{ \pm}^{2} T M$. If $\mathcal{Z}$ is the twistor space of $(M, g)$, we can define four almost complex structures $g^{m}$ on the product bundle $\mathcal{Z} \times \mathcal{Z} \rightarrow M$ (the generalized twistor space) as follows. If $J=\left(J_{1}, J_{2}\right) \in \mathcal{Z} \times \mathcal{Z}$, the horizontal space $\mathcal{H}_{J}$ at $J$ of the vector bundle $\Lambda^{2} T M \times \Lambda^{2} T M$ with respect to the connection $D$ is tangent to $\mathcal{Z} \times \mathcal{Z}$, and we set $g^{m} X_{J}^{h}=\left(J_{1} X\right)_{J}^{h}$ for every $X \in T_{\pi(J)} M$ and $m=1, \ldots, 4$. There are four natural complex structures on the vertical space $\mathcal{V}_{J}=\mathcal{V}_{J_{1}} \times \mathcal{V}_{J_{2}}$ of $\mathcal{Z} \times \mathcal{Z}$ defined for $V=\left(V_{1}, V_{2}\right)$ by $K_{1} V=\left(J_{1} V_{1}, J_{2} V_{2}\right), K_{2} V=\left(J_{1} V_{1},-J_{2} V_{2}\right)$, $K_{3} V=-K_{2} V, K_{4} V=-K_{1} V$. We set $g^{m} V=K_{m} V, m=1, \ldots, 4$.

Remark. In order to define a complex structure on the horizontal spaces $\mathcal{H}_{J}, J=$ $\left(J_{1}, J_{2}\right)$, we can use the structure $J_{2}$ instead of $J_{1}$. Then we get four more almost complex structures on $\mathcal{Z} \times \mathcal{Z}$. But, by symmetry, they do not differ essentially from the structures $\mathrm{g}^{m}, m=1, \ldots, 4$.

In this section, we shall find the integrability conditions for the restrictions of $g^{m}$ to the four connected components of $\mathcal{Z} \times \mathcal{Z}$, the subbundles $\mathcal{Z}_{ \pm} \times \mathcal{Z}_{ \pm}$, under the assumption that the torsion of $D$ is skew-symmetric.

Denote by $\mathcal{N}_{m}$ the Nijenhuis tensor of the almost complex structure $\mathfrak{f}^{m}$. Then we have the following analog of Lemma 1 (with a similar proof).

Proposition 1. Let $J=\left(J_{1}, J_{2}\right) \in \mathcal{Z} \times \mathcal{Z}, V=\left(V_{1}, V_{2}\right), W \in \mathcal{V}_{J}$, and let $X, Y \in$ $T_{\pi(J)} M$. Then

(i) $\mathcal{H} \mathcal{N}_{m}\left(X^{h}, Y^{h}\right)_{J}=\left(T(X, Y)-T\left(J_{1} X, J_{1} Y\right)+J_{1} T\left(J_{1} X, Y\right)+J_{1} T\left(X, J_{1} Y\right)\right)_{J}^{h}$.

(ii) $\mathcal{V} \mathcal{N}_{m}\left(X^{h}, Y^{h}\right)_{J}=-R^{D}(X, Y) J+R^{D}\left(J_{1} X, J_{1} Y\right) J$

$$
-K_{m}\left(R^{D}\left(J_{1} X, Y\right) J+R^{D}\left(X, J_{1} Y\right) J\right) .
$$

(iii) $\mathcal{N}_{m}\left(X_{J}^{h}, V\right)=0 \quad$ for $\quad m=1,2$.

$\mathcal{N}_{m}\left(X_{J}^{h}, V\right)=2\left(J_{1} V_{1} X\right)_{J}^{h} \quad$ for $\quad m=3,4$.

(iv) $\mathcal{N}_{m}(V, W)=0$.

Corollary 2. The restrictions of the almost complex structures $\mathrm{J}^{m}, m=3,4$, to the connected components of $\mathcal{Z} \times \mathcal{Z}$ are not integrable. 
In order to determine the integrability conditions for $\mathcal{J}^{1}$ and $\mathcal{J}^{2}$, we need the following.

Notation. The 1-form $* \mathcal{T}$ will be denoted by $\tau$.

Clearly, the form $\tau$ uniquely determines the 3 -form $\mathcal{T}$, hence the connection $D$.

For a given orthonormal frame $E_{1}, \ldots, E_{4}$, it is convenient to set

$$
E_{i j k}=E_{i} \wedge E_{j} \wedge E_{k}, \quad \mathcal{T}_{i j k}=\mathcal{T}\left(E_{i j k}\right) .
$$

Under this notation, we have

$$
\begin{gathered}
\mathcal{T}=\mathcal{T}_{123} E_{123}+\mathcal{T}_{124} E_{124}+\mathcal{T}_{134} E_{134}+\mathcal{T}_{234} E_{234} \\
\tau=-\mathcal{T}_{234} E_{1}+\mathcal{T}_{134} E_{2}-\mathcal{T}_{124} E_{3}+\mathcal{T}_{123} E_{4} .
\end{gathered}
$$

It follows that

$$
\begin{array}{ll}
\left(\nabla_{X} \mathcal{T}\right)\left(E_{123}\right)=\left(\nabla_{X} \tau\right)\left(E_{4}\right), & \left(\nabla_{X} \mathcal{T}\right)\left(E_{124}\right)=-\left(\nabla_{X} \tau\right)\left(E_{3}\right) \\
\left(\nabla_{X} \mathcal{T}\right)\left(E_{134}\right)=\left(\nabla_{X} \tau\right)\left(E_{2}\right), & \left(\nabla_{X} \mathcal{T}\right)\left(E_{234}\right)=-\left(\nabla_{X} \tau\right)\left(E_{1}\right)
\end{array}
$$

Theorem 2. The restriction of the almost complex structure $\mathrm{g}^{m}, m=1$ or 2 , to $\mathcal{Z}_{+} \times \mathcal{Z}_{+}$(respectively, $\mathcal{Z}_{-} \times \mathcal{Z}_{-}$) is integrable if and only if the metric $g$ is antiself-dual (resp. self-dual) with scalar curvature $s=\frac{3}{2}\|\tau\|^{2}+3 \delta \tau$ and the 2 -form $d \tau$ is anti-self-dual (resp. self-dual).

Proof. According to Proposition 1 and Lemma 2, the almost complex structure $\mathrm{J}^{m}$, $m=1$ or 2 , on $\mathcal{Z}_{+} \times \mathcal{Z}_{+}$is integrable if and only for every $J=\left(J_{1}, J_{2}\right) \in \mathcal{Z}_{+} \times \mathcal{Z}_{+}$ and every $X, Y, Z, U \in T_{p} M, p=\pi(J)$,

$$
\begin{aligned}
& g\left(\mathcal{R}^{D}\left(X \wedge Y-J_{1} X \wedge J_{1} Y\right), Z \wedge U-J_{i} Z \wedge J_{i} U\right) \\
= & \pm g\left(\mathcal{R}^{D}\left(J_{1} X \wedge Y+X \wedge J_{1} Y\right), J_{i} Z \wedge U+Z \wedge J_{i} U\right), \quad i=1,2,
\end{aligned}
$$

where the minus sign corresponds to the case $m=i=2$.

By Lemmas 1 and 2 the latter identity for $i=1$ is the integrability condition for the Atiyah-Hitchin-Singer almost complex structure on $\mathcal{Z}_{+}$.

Now, we show that the identity (9) with $i=2$ is equivalent to

$$
g\left(\mathcal{R}^{D}(a), b\right)=0 \text { for every } a, b \in \Lambda_{+}^{2} T M .
$$

Suppose that

$$
\begin{aligned}
& g\left(\mathcal{R}^{D}\left(X \wedge Y-J_{1} X \wedge J_{1} Y\right), Z \wedge U-J_{2} Z \wedge J_{2} U\right) \\
& =(-1)^{m+1} g\left(\mathcal{R}^{D}\left(J_{1} X \wedge Y+X \wedge J_{1} Y\right), J_{2} Z \wedge U+Z \wedge J_{2} U\right)
\end{aligned}
$$

for every $\left(J_{1}, J_{2}\right)$ and every $X, Y, Z, U, m=1,2$. Let $J \in \mathcal{Z}_{+}$be a complex structure on a tangent space $T_{p} M$. Applying (11) with $\left(J_{1}, J_{2}\right)=(J, J)$ and $\left(J_{1}, J_{2}\right)=$ $(J,-J)$, we see that in the both cases $m=1$ and $m=2$

$$
g\left(\mathcal{R}^{D}(X \wedge Y-J X \wedge J Y), Z \wedge U-J Z \wedge J U\right)=0
$$

for every $X, Y, Z, U \in T_{p} M$. Let $\left(E_{1}, \ldots, E_{4}\right)$ be an oriented orthonormal basis of $T_{p} M$, and define $s_{i}=s_{i}^{+}$by means of this basis. Setting $J=s_{1},(X, Y)=$ $\left(E_{1}, E_{3}\right),(Z, U)=\left(E_{1}, E_{3}\right)$ in (12), we get the identity $g\left(R^{D}\left(s_{2}\right), s_{2}\right)=0$; for 
$(Z, U)=\left(E_{1}, E_{4}\right)$, we obtain $g\left(R^{D}\left(s_{2}\right), s_{3}\right)=0$. Also, identity (12) with $J=s_{2}$, $(X, Y)=\left(E_{1}, E_{3}\right)$, and $(Z, U)=\left(E_{1}, E_{2}\right)$ implies $g\left(R^{D}\left(s_{2}\right), s_{1}\right)=0$. Thus,

$$
g\left(R^{D}\left(s_{2}\right), s_{1}\right)=g\left(R^{D}\left(s_{2}\right), s_{2}\right)=g\left(R^{D}\left(s_{2}\right), s_{3}\right)=0 .
$$

Applying the latter identities for the bases $\left(E_{1}, E_{4}, E_{2}, E_{3}\right)$ and $\left(E_{1}, E_{3}, E_{4}, E_{2}\right)$, we see that

$$
\begin{aligned}
& g\left(R^{D}\left(s_{1}\right), s_{1}\right)=g\left(R^{D}\left(s_{1}\right), s_{2}\right)=g\left(R^{D}\left(s_{1}\right), s_{3}\right)=0, \\
& g\left(R^{D}\left(s_{3}\right), s_{1}\right)=g\left(R^{D}\left(s_{3}\right), s_{2}\right)=g\left(R^{D}\left(s_{3}\right), s_{3}\right)=0 .
\end{aligned}
$$

Identities (13) and (14) clearly imply (10). This shows that (10) follows from (11).

In order to show that (10) implies (11), it is enough to note that if $J \in \mathcal{Z}_{+}$and $X, Y \in T_{\pi(J)} M$, then the 2-vector $X \wedge Y-J X \wedge J Y \in \Lambda_{+}^{2} T_{\pi(J)} M$. This follows from the easily verifying fact that $X \wedge Y-J X \wedge J Y$ is orthogonal to $\Lambda_{-}^{2} T_{p} M$, $p=\pi(J)$, since $J$ commutes with every endomorphism of $T_{p} M$ lying in $\Lambda_{-}^{2} T_{p} M$.

The components of the curvature operator $\mathcal{R}^{D}: \Lambda^{2} T M \rightarrow \Lambda^{2} T M$ with respect to the decomposition $\Lambda^{2} T M=\Lambda_{+}^{2} T M \oplus \Lambda_{-}^{2} T M$ have been computed in [12]. In fact, using (6), (7) and (8), we compute

$$
\begin{aligned}
& g\left(\mathcal{R}^{D}\left(s_{1}\right), s_{1}\right)=g\left(R^{D}\left(E_{1}, E_{2}\right) E_{1}, E_{2}\right)+g\left(R^{D}\left(E_{1}, E_{2}\right) E_{3}, E_{4}\right) \\
& +g\left(R^{D}\left(E_{3}, E_{4}\right) E_{1}, E_{2}\right)+g\left(R^{D}\left(E_{3}, E_{4}\right) E_{3}, E_{4}\right) \\
& =-\frac{1}{4}\left[\mathcal{T}\left(E_{1}, E_{2}, E_{3}\right)^{2}+\mathcal{T}\left(E_{1}, E_{2}, E_{4}\right)^{2}\right]-\frac{1}{2}\left[\left(\nabla_{E_{1}} \mathcal{T}\right)\left(E_{2}, E_{3}, E_{4}\right)-\left(\nabla_{E_{2}} \mathcal{T}\right)\left(E_{3}, E_{4}, E_{1}\right)\right] \\
& -\frac{1}{2}\left[\left(\nabla_{E_{3}} \mathcal{T}\right)\left(E_{4}, E_{1}, E_{2}\right)-\left(\nabla_{E_{4}} \mathcal{T}\right)\left(E_{1}, E_{2}, E_{3}\right)\right]-\frac{1}{4}\left[\mathcal{T}\left(E_{3}, E_{4}, E_{1}\right)^{2}+\mathcal{T}\left(E_{3}, E_{4}, E_{2}\right)^{2}\right] \\
& +g\left(R^{\nabla}\left(s_{1}\right), s_{1}\right) \\
& =-\frac{1}{4}\|\tau\|^{2}-\frac{1}{2} \delta \tau+g\left(R^{\nabla}\left(s_{1}\right), s_{1}\right) \text {. }
\end{aligned}
$$

A similar computation gives

$$
g\left(\mathcal{R}^{D}\left(s_{1}\right), s_{2}\right)=\frac{1}{2}(d \tau)\left(s_{3}\right)+g\left(\mathcal{R}^{\nabla}\left(s_{1}\right), s_{2}\right) .
$$

Also,

$$
g\left(\mathcal{R}^{D}\left(s_{1}\right), s_{3}\right)=-\frac{1}{2}(d \tau)\left(s_{2}\right)+g\left(\mathcal{R}^{\nabla}\left(s_{1}\right), s_{3}\right) .
$$

Replacing the basis $\left(E_{1}, E_{2}, E_{2}, E_{4}\right)$ by $\left(E_{1}, E_{3}, E_{4}, E_{2}\right)$ and $\left(E_{1}, E_{4}, E_{2}, E_{3}\right)$, we see that

$$
\begin{gathered}
g\left(\mathcal{R}^{D}\left(s_{i}\right), s_{i}\right)=-\frac{1}{4}\|\tau\|^{2}-\frac{1}{2} \delta \tau+g\left(R^{\nabla}\left(s_{i}\right), s_{i}\right), \quad i=1,2,3, \\
g\left(\mathcal{R}^{D}\left(s_{i}\right), s_{j}\right)=\frac{1}{2}(d \tau)\left(s_{i} \times s_{j}\right)+g\left(\mathcal{R}^{\nabla}\left(s_{i}\right), s_{j}\right), \quad i \neq j .
\end{gathered}
$$

Now, suppose that the structure $\mathcal{J}^{m}$ is integrable, $m=1$ or 2 . Then the manifold $(M, g)$ is anti-self-dual by Theorem 1, and identities (10), (15), and (5) imply

$$
-\frac{1}{4}\|\tau\|^{2}-\frac{1}{2} \delta \tau+\frac{s}{6}=0, \quad(d \tau) \mid \Lambda_{+}^{2} T M=0 .
$$

The latter identity means that $* d \tau=-d \tau$, i.e., the 2 -form $d \tau$ is anti-self-dual.

Conversely, suppose that identities (16) are satisfied and the metric $g$ is anti-selfdual. Then (15) implies (10), hence the almost complex structure $f^{m}$ is integrable on $\mathcal{Z}_{+} \times \mathcal{Z}_{+}$. 
Changing the orientation of $M$, we obtain the integrability condition for the restriction of $\mathcal{g}^{m}$ to $\mathcal{Z}_{-} \times \mathcal{Z}_{-}$.

Remarks 1. In the case when $\tau=0$, i.e., $D$ is the Levi-Civita connection, Theorem 2 coincides with [9, Theorem 2 (a) and (b)]

2. As is well-known, if $M$ is compact, the condition that $d \tau$ is anti-self-dual implies $d \tau=0$ by integrating the identity $d(d \tau \wedge \tau)=-d \tau \wedge \star d \tau=-\|d \tau\|^{2}$ vol.

3. The identity

$$
s=\frac{3}{2}\|\tau\|^{2}+3 \delta \tau
$$

can be interpreted in terms of the Weyl geometry. Recall that a Weyl connection on a conformal $n$-dimensional manifold is a torsion-free connection $\nabla^{w}$ which preserves the conformal structure. This means that for every Riemannian metric $g$ in the conformal class there exists a 1 -form $\theta_{g}$ such that $\nabla^{w} g=\theta_{g} \otimes g$; obviously, such a form is unique. If $\nabla^{g}$ is the Levi-Civita connection of the metric $g$,

$$
\nabla_{X}^{w} Y=\nabla_{X}^{g} Y-\frac{1}{2}\left[\theta_{g}(X) Y+\theta_{g}(Y) X-g(X, Y) \theta_{g}^{\sharp}\right],
$$

where $\theta_{g}^{\sharp}$ is the dual vector field of the form $\theta_{g}$ with respect to the metric $g$, $g\left(\theta^{\sharp}, Z\right)=\theta_{g}(Z)$. If $\widetilde{g}=e^{f} g$ is another Riemannian metric in the conformal class, where $f$ is a smooth function, $\theta_{\widetilde{g}}=d f+\theta_{g}$. Hence the condition $d \theta_{g}=0$ does not depend on the choice of the metric $g$. In this case, we say that $\nabla^{w}$ determines a closed Weyl structure. If $d \theta_{g}=0$, then locally $\theta_{g}=d \psi$ for a smooth function $\psi$, so $\nabla^{w}$ concides locally with the Levi-Civita connection of the metric $e^{-\psi} g$. The condition that the form $\theta_{g}$ is exact also does not depend on the choice of $g$, and a Weyl structure with exact $\theta_{g}$ is called exact.

It follows from (18) that the Ricci tensors $R i c^{w}$ and $R i c^{g}$ of the connections $\nabla^{w}$ and $\nabla^{g}$ are related by

$$
\begin{aligned}
\operatorname{Ric}^{w}(X, Y)= & \operatorname{Ric}^{g}(X, Y)+\frac{n-1}{2}\left(\nabla_{X}^{g} \theta_{g}\right)(Y)-\frac{1}{2}\left(\nabla_{Y}^{g} \theta_{g}\right)(X) \\
& -\frac{n-2}{4}\left[\left\|\theta_{g}\right\|^{2} g(X, Y)-\theta_{g}(X) \theta_{g}(Y)\right]-\frac{1}{2}\left(\delta^{g} \theta_{g}\right) g(X, Y),
\end{aligned}
$$

where the norm and the codifferential are taken with respect to the metric $g$. Hence the symmetric part $\operatorname{Ric}^{s y m}=\frac{1}{2}\left[\operatorname{Ric}^{w}(X, Y)+\operatorname{Ric}^{w}(Y, X)\right]$ of the Ricci tensor of the connection $\nabla^{w}$ is

$$
\begin{aligned}
\operatorname{Ric}^{\text {sym }}(X, Y)= & \operatorname{Ric}^{g}(X, Y)+\frac{n-2}{2}\left[\left(\nabla_{X}^{g} \theta_{g}\right)(Y)+\left(\nabla_{Y}^{g} \theta_{g}\right)(X)\right] \\
& \left.-\frac{n-2}{4}\left[\left\|\theta_{g}\right\|^{2} g(X, Y)-\theta_{g}(X) \theta_{g}(Y)\right]-\frac{1}{2}\left(\delta^{g} \theta_{g}\right) g(X, Y)\right] .
\end{aligned}
$$

Therefore, if $s^{g}$ is the scalar curvature of $g$, the trace of $R i c^{s y m}$ with respect to $g$ is

$$
s_{g}^{w}=s^{g}-\frac{(n-1)(n-2)}{4}\left\|\theta_{g}\right\|^{2}-(n-1) \delta^{g} \theta_{g} .
$$

Clearly, if $\widetilde{g}=e^{f} g$, then $s_{\widetilde{g}}^{w}=e^{-f} s_{g}^{w}$. The function $s_{g}^{w}$ is called the conformal scalar curvature with respect to $g$. Its vanishing does not depend on the choice of the metric $g$ in the conformal class. We note also that $s_{g}^{w}$ represents a section of the so-called line bundle of weight -2 , see, for example, [5, 14. 
Now, consider the conformal structure on the Riemannian 4- manifold $M$ determined by the metric $g$. Define a connection $\nabla^{w}$ on $M$ by (18) with $\theta_{g}=\tau$. Then $\nabla^{w}$ is a Weyl connection and the identity (17) is equivalent to the vanishing of the conformal scalar curvature.

As is well-known, the Weyl tensor is conformally invariant, hence so are the conditions $\mathcal{W}_{+}=0$ and $\mathcal{W}_{-}=0$. Therefore, if $(M, g, \tau)$ satisfies the conditions of Theorem 2 and $f$ is a smooth function on $M$, then $\left(M, e^{f} g, d f+\tau\right)$ also satisfies these conditions.

As we have noticed, $d \tau=0$ in the case of a compact manifold $M$. Suppose that $\tau=d f$ for a smooth function $f$ on $M$. Let $\widetilde{\delta}$ be the codifferential with respect to the metric $\widetilde{g}=e^{-2 f} g$. Using the well-known formula $\widetilde{\delta} \tau=e^{2 f}(\delta \tau+2 \tau(\operatorname{grad} f))$, we see that if $\tau$ satisfies (17),

$$
e^{2 f} s=-\frac{9}{2} e^{2 f}\|d f\|_{g}^{2}+3 \widetilde{\delta} d f .
$$

Hence, if $\widetilde{\mu}$ is the volume form with respect to the metric $\widetilde{g}$,

$$
\int_{M} e^{2 f} s \widetilde{\mu}=-\frac{9}{2} \int_{M} e^{2 f}\|d f\|_{g}^{2} \widetilde{\mu} \leq 0 .
$$

Also, if $\mu$ is the volume form with respect to the metric $g$, it follows from (17) that

$$
\int_{M} s \mu=\frac{3}{2} \int_{M}\|\tau\|_{g}^{2} \mu \geq 0 .
$$

Therefore either $s \equiv 0$ or $s$ takes values with different signs. Thus, if $H^{1}(M, \mathbb{R})=0$ and $s=$ const $\neq 0$, there does not exist 1-form $\tau$ satisfying (17). In particular, this holds for $M=S^{4}$ and $M=\mathbb{C P}^{2}$.

Examples. 1. Let $(M, g, J)$ be a Hermitian surface with fundamental 2-form $\Omega(X, Y)=g(X, J Y)$ and Lee from $\theta=\delta \Omega \circ J$. Denote by $s$ and $s^{*}$ the scalar and *-scalar curvatures. Then, by [30, Theorem 3.1],

$$
s-s^{*}=\|\theta\|^{2}+2 \delta \theta .
$$

According to [20, Proposition 6.4], a Hermitian surface is anti-self-dual if and only if $s=3 s^{*}$ and the 2-form $d \theta$ is anti-self-dual. Therefore the Lee form of an antiself-dual Hermitian surface satisfies the conditions of Theorem 2 with $\tau=\theta$. Apparently, if the surface is Kähler, $\theta=0$. Note that, as is well-known, a Kähler surface is anti-self-dual if and only if it is scalar-flat. In particular, by the famous Yau's solution of the Calabi conjecture, every compact Kähler surface with vanishing first Chern class admits an anti-self-dual Kähler metric. There are many other constructions of scalar-flat Kähler surfaces and we refer to LeBrun's survey paper [23. and the literature therein, as well as to [21, 19, 27, for results in this area. The standard Hermitian structure on the Hopf surface $S^{3} \times S^{1}$ is non-Kähler, conformally flat $\left(\mathcal{W}_{+}=\mathcal{W}_{-}=0\right)$ and locally conformally Kähler $(d \theta=0)$, so it satisfies the conditions of Theorem 2, C. LeBrun [22] has constructed non-Kähler anti-self-dual Hermitian metrics on the blow-ups $\left(S^{3} \times S^{1}\right) \sharp n \overline{\mathbb{C P}^{2}}$ of the Hopf surface. I. Kim [18 has shown the existence of anti-self-dual strictly almost Kähler structures on $\mathbb{C P}^{2} \sharp n \overline{\mathbb{C P}^{2}}, n \geq 11,\left(S^{2} \times \Sigma\right) \sharp n \overline{\mathbb{C P}^{2}},\left(S^{2} \times T^{2}\right) \sharp n \overline{\mathbb{C P}^{2}}, n \geq 6$, where $\Sigma$ is a compact Riemann surface of genus $\geq 2$ and $T^{2}$ is the torus. 
2. Following [29], consider a real 4-dimensional vector space $V$ oriented by a basis $\left(E_{1}, \ldots, E_{4}\right)$. For $\lambda \in \mathbb{R}$, let $\mathfrak{g}_{\lambda}$ be the Lie algebra on $V$ defined by the relations

$$
\left[E_{1}, E_{2}\right]=E_{2}-\lambda E_{3}, \quad\left[E_{1}, E_{3}\right]=\lambda E_{2}+E_{3}, \quad\left[E_{1}, E_{4}\right]=2 E_{4}, \quad\left[E_{2}, E_{3}\right]=-E_{4},
$$

and the other brackets equal zero. It is easy to check that this algebra is solvable.

It is shown in 29 that $\mathfrak{g}_{\lambda}$ and $\mathfrak{g}_{\lambda^{\prime}}$ are not isomorphic if $\lambda^{\prime} \neq \pm \lambda$. The algebras $\mathfrak{g}_{\lambda}$ and $\mathfrak{g}_{-\lambda}$ are isomorphic by the map which just changes the signs of $E_{3}$ and $E_{4}$.

Let $G_{\lambda}$ be the simply connected Lie group corresponding to $\mathfrak{g}_{\lambda}$. This group is not compact. Otherwise, it would admit an inner product such that all endomorphisms $a d_{X}, X \in \mathfrak{g}_{\lambda}$, were skew-symmetric. Hence $E_{4}$ would have zero length since $a d_{E_{1}}\left(E_{4}\right)=2 E_{4}$, a contradiction. Note also that $\operatorname{Trace} a d_{E_{1}} \neq 0$, so the group $G_{\lambda}$ is not unimodular, hence it does not admit a co-compact lattice.

For $k>0$, let $g_{k}$ be the left invariant metric on $G_{\lambda}$ for which $\left(\frac{1}{k} E_{1}, E_{2}, E_{3}, E_{4}\right)$ is an orthonormal basis. It is proved in 29 that the Riemannian manifolds $\left(G_{\lambda}, g_{k}\right)$ and $\left(G_{\lambda^{\prime}}, g_{k^{\prime}}\right)$ are isometric if and only if $k=k^{\prime}$. Hence the curvature of $\left(G_{\lambda}, g_{k}\right)$ does not depend on $\lambda$. According to [29, the Ricci tensor of $g_{k}$ is given by

$$
\operatorname{Ric}\left(E_{1}, E_{1}\right)=6, \quad \operatorname{Ric}\left(E_{2}, E_{2}\right)=\operatorname{Ric}\left(E_{3}, E_{3}\right)=\frac{4}{k^{2}}+\frac{1}{2}, \quad \operatorname{Ric}\left(E_{4}, E_{4}\right)=\frac{8}{k^{2}}-\frac{1}{2},
$$

and $\operatorname{Ric}\left(E_{i}, E_{j}\right)=0$ for $i \neq j$. Hence the scalar curvature of $g_{k}$ is

$$
s_{k}=\frac{22}{k^{2}}+\frac{1}{2}
$$

Also, the two halves of the Weyl operator are computed in [29]. The result there shows that $\mathcal{W}_{+}=0$ if and only if $k \in\{1,2\}$ and, for these values of $k, \mathcal{W}_{-} \neq 0$. The orientation of $G_{\lambda}$ is, of course, just a matter of choice and changing the orientation interchanges the roles of $\mathcal{W}_{+}$and $\mathcal{W}_{-}$.

It is proved in 29 that if $G$ is an oriented four-dimensional Lie group admitting a left invariant Riemannian metric $g$ such that $\mathcal{W}_{+}=0$ and $\mathcal{W}_{-} \neq 0$, then the Lie algebra of $G$ is isomorphic to $\mathfrak{g}_{\lambda}$ for some $\lambda \geq 0$ and $g$ is locally homothetic to either $g_{1}$ or $g_{2}$.

Extend $\left(E_{1}, \ldots, E_{4}\right)$ to a frame of left-invariant vector fields on $G_{\lambda}$. Let $\left(\alpha^{1}, \ldots, \alpha^{4}\right)$ be the dual frame to $\left(E_{1}, \ldots, E_{4}\right)$. Then

$$
\begin{gathered}
d \alpha^{1}=0, \quad d \alpha^{2}=-\alpha^{1} \wedge \alpha^{2}-\lambda \alpha^{1} \wedge \alpha^{3}, \\
d \alpha^{3}=\lambda \alpha^{1} \wedge \alpha^{2}-\alpha^{1} \wedge \alpha^{3}, \quad d \alpha^{4}=-2 \alpha^{1} \wedge \alpha^{4}+\alpha^{2} \wedge \alpha^{3} .
\end{gathered}
$$

Let $\tau=\sum_{i=1}^{4} \mu_{i} \alpha^{i}, \mu_{i} \in \mathbb{R}$, be a left invariant 1 -form on $G_{\lambda}$. Then, if $\delta^{k}$ is the codifferential with respect to the metric $g_{k}, \delta^{k} \tau=\frac{4}{k^{2}} \mu_{1}$. Let $s_{1}^{+}, s_{2}^{+}, s_{3}^{+}$be the basis of $\Lambda_{+}^{2} \mathfrak{g}_{\lambda}$ defined via (11) by means of the orthonormal basis $\left(\frac{1}{k} E_{1}, E_{2}, E_{3}, E_{4}\right)$, where $k=1$ or $k=2$. The identities $d \tau\left(s_{i}^{+}\right)=0$ for $i=1,2,3$ are equivalent to $\mu_{2}=\mu_{3}=\mu_{4}$ in the case $k=1$ and $\mu_{2}=\mu_{3}=0$ when $k=2$. Thus, if $k=1$, identity (17) is satisfied for $\mu_{1}=-4 \pm \sqrt{31}$; if $k=2$, it is satisfied iff $\mu_{1}$ and $\mu_{4}$ are related by $\mu_{1}^{2}+8 \mu_{1}-16+4 \mu_{4}^{2}=0$.

Theorem 3. The almost complex structure $\mathcal{J}^{m}$ on $\mathcal{Z}_{+} \times \mathcal{Z}_{-}$(resp. $\left.\mathcal{Z}_{-} \times \mathcal{Z}_{+}\right), m=1$ or 2 , is integrable if and only if the metric $g$ is anti-self-dual (resp. self-dual) and its Ricci tensor $\rho$ is given by

$$
\rho(X, Y)=\left[\mathcal{S}(\nabla \tau)-\frac{1}{2} \tau \otimes \tau+\frac{1}{8}\left(2 s+2 \delta \tau+\|\tau\|^{2}\right) g\right](X, Y),
$$


where $\mathcal{S}$ stands for the symmetrization of a bilinear form.

Proof. By Proposition 1 and Lemma 2, the almost complex structure $\mathfrak{J}^{m}$ on $\mathcal{Z}_{+} \times \mathcal{Z}_{-}$ is integrable if and only the metric $g$ is anti-self-dual and for every $J=\left(J_{1}, J_{2}\right) \in$ $\mathcal{Z}_{+} \times \mathcal{Z}_{-}$

$$
\begin{aligned}
& g\left(\mathcal{R}^{D}\left(X \wedge Y-J_{1} X \wedge J_{1} Y\right), Z \wedge U-J_{2} Z \wedge J_{2} U\right) \\
& =(-1)^{m+1} g\left(\mathcal{R}^{D}\left(J_{1} X \wedge Y+X \wedge J_{1} Y\right), J_{2} Z \wedge U+Z \wedge J_{2} U\right)
\end{aligned}
$$

for every $X, Y, Z, U \in T_{\pi(J)} M, m=1,2$.

Let $\left(E_{1}, \ldots, E_{4}\right)$ be an oriented orthonormal basis of a tangent space $T_{p} M$, and define $\left\{s_{i}^{ \pm}\right\}$via (1). Then identity (22) is equivalent to

$$
g\left(R^{D}\left(s_{i}^{+}\right), s_{j}^{-}\right)=0, \quad i, j=1,2,3 .
$$

Indeed, if the latter identity holds, the identity (22) holds as well since $A \wedge B-$ $J_{1} A \wedge J_{1} B \in \Lambda_{+}^{2} T_{\pi(J)} M, A \wedge B-J_{2} A \wedge J_{2} B \in \Lambda_{-}^{2} T_{\pi(J)} M$ for every $A, B \in T_{\pi(J)} M$. Conversely, suppose that (22) is satisfied. Then this identity with $J_{1}=s_{1}^{+}, J_{2}=$ $(-1)^{m+1} s_{1}^{-},(X, Y)=(Z, U)=\left(E_{1}, E_{3}\right)$ gives

$$
g\left(R^{D}\left(s_{2}^{+}\right), s_{2}^{-}\right)=-g\left(R^{D}\left(s_{3}^{+}\right), s_{3}^{-}\right) .
$$

Replacing the basis $\left(E_{1}, E_{2}, E_{3}, E_{4}\right)$ by $\left(E_{1}, E_{3}, E_{4}, E_{2}\right)$ and $\left(E_{1}, E_{4}, E_{2}, E_{3}\right)$, we get from the identity above

$$
g\left(R^{D}\left(s_{3}^{+}\right), s_{3}^{-}\right)=-g\left(R^{D}\left(s_{1}^{+}\right), s_{1}^{-}\right), \quad g\left(R^{D}\left(s_{1}^{+}\right), s_{1}^{-}\right)=-g\left(R^{D}\left(s_{2}^{+}\right), s_{2}^{-}\right) .
$$

It follows that

$$
g\left(R^{D}\left(s_{i}^{+}\right), s_{i}^{-}\right)=0, \quad i=1,2,3 .
$$

Identity (22) with $J_{1}=s_{1}^{+},(X, Y)=\left(E_{1}, E_{4}\right), J_{2}=(-1)^{m+1} s_{3}^{-},(Z, U)=\left(E_{1}, E_{2}\right)$ implies $g\left(R^{D}\left(s_{3}^{+}\right), s_{1}^{-}\right)=g\left(R^{D}\left(s_{2}^{+}\right), s_{2}^{-}\right)$. Setting $J_{1}=s_{2}^{+},(X, Y)=\left(E_{1}, E_{4}\right)$, $J_{2}=(-1)^{m+1} s_{3}^{-},(Z, U)=\left(E_{1}, E_{3}\right)$, we also get $g\left(R^{D}\left(s_{3}^{+}\right), s_{2}^{-}\right)=g\left(R^{D}\left(s_{1}^{+}\right), s_{1}^{-}\right)$. Thus,

$$
g\left(R^{D}\left(s_{3}^{+}\right), s_{1}^{-}\right)=g\left(R^{D}\left(s_{3}^{+}\right), s_{2}^{-}\right)=0 .
$$

Again replacing the basis $\left(E_{1}, E_{2}, E_{3}, E_{4}\right)$ by the bases $\left(E_{1}, E_{3}, E_{4}, E_{2}\right)$ and $\left(E_{1}, E_{4}, E_{2}, E_{3}\right)$, we see that

$$
g\left(R^{D}\left(s_{i}^{+}\right), s_{j}^{-}\right)=0, \quad i \neq j .
$$

Now, using (6), we compute

$$
\begin{aligned}
g\left(\mathcal{R}^{D}\left(s_{1}^{+}\right), s_{1}^{-}\right)= & \left.\frac{1}{2}\left[\left(\nabla_{E_{1}} \mathcal{T}\right)\left(E_{234}\right)-\left(\nabla_{E_{2}} \mathcal{T}\right)\left(E_{134}\right)-\left(\nabla_{E_{3}} \mathcal{T}\right)\left(E_{124}\right)\right)+\left(\nabla_{E_{4}} \mathcal{T}\right)\left(E_{123}\right)\right] \\
& +\frac{1}{4}\left[-\mathcal{T}_{123}^{2}-\mathcal{T}_{124}^{2}+\mathcal{T}_{134}^{2}+\mathcal{T}_{234}^{2}\right]+g\left(\mathcal{R}^{\nabla}\left(s_{1}^{+}\right), s_{1}^{-}\right) .
\end{aligned}
$$

Moreover, setting $\rho_{i j}=\rho\left(E_{i}, E_{j}\right)$, we have by (44)

$$
g\left(\mathcal{R}^{\nabla}\left(s_{1}^{+}\right), s_{1}^{-}\right)=g\left(\mathcal{B}\left(s_{1}^{+}\right), s_{1}^{-}\right)=\frac{1}{2}\left[\rho_{11}+\rho_{22}-\rho_{33}-\rho_{44}\right] .
$$

Thus, taking into account (8), we obtain

$$
\begin{gathered}
\left.g\left(\mathcal{R}^{D}\left(s_{1}^{+}\right), s_{1}^{-}\right)=\frac{1}{2}\left[-\left(\nabla_{E_{1}} \tau\right)\left(E_{1}\right)-\left(\nabla_{E_{2}} \tau\right)\left(E_{2}\right)+\left(\nabla_{E_{3}} \tau\right)\left(E_{3}\right)\right)+\left(\nabla_{E_{4}} \tau\right)\left(E_{4}\right)\right] \\
+\frac{1}{4}\left[-\tau_{4}^{2}-\tau_{3}^{2}+\tau_{2}^{2}+\tau_{1}^{2}\right]+\frac{1}{2}\left[\rho_{11}+\rho_{22}-\rho_{33}-\rho_{44}\right]
\end{gathered}
$$


where $\tau_{i}=\tau\left(E_{i}\right), i=1, . ., 4$. Applying the latter identity for the bases $\left(E_{1}, E_{3}, E_{4}, E_{2}\right)$ and $\left(E_{1}, E_{4}, E_{2}, E_{3}\right)$, we get

$$
0=4 \sum_{i=1}^{3} g\left(\mathcal{R}^{D}\left(s_{i}^{+}\right), s_{i}^{-}\right)=2\left[-4\left(\nabla_{E_{1}} \tau\right)\left(E_{1}\right)-\delta \tau\right]-\|\tau\|^{2}+4 \tau_{1}^{2}+8 \rho_{11}-2 s .
$$

For a bilinear form $\alpha$, let $(\mathcal{S} \alpha)(X, Y)=\frac{1}{2}[\alpha(X, Y)+\alpha(Y, X)]$ be the symmetrization of $\alpha$. Then the latter identity can be written as

$$
\rho\left(E_{1}, E_{1}\right)=\left[\mathcal{S}(\nabla \tau)-\frac{1}{2} \tau \otimes \tau+\frac{1}{8}\left(2 s+2 \delta \tau+\|\tau\|^{2}\right) g\right]\left(E_{1}, E_{1}\right) .
$$

Moreover,

$$
\begin{gathered}
0=g\left(\mathcal{R}^{D}\left(s_{1}^{+}\right), s_{2}^{-}\right)+g\left(\mathcal{R}^{D}\left(s_{2}^{+}\right), s_{1}^{-}\right)=\left(\nabla_{E_{2}} \mathcal{T}\right)\left(E_{124}\right)-\left(\nabla_{E_{3}} \mathcal{T}\right)\left(E_{134}\right)-\mathcal{T}_{124} \mathcal{T}_{134} \\
+g\left(\mathcal{B}\left(s_{1}^{+}\right), s_{2}^{-}\right)+g\left(\mathcal{B}\left(s_{2}^{+}\right), s_{1}^{-}\right) \\
=-\left(\nabla_{E_{2}} \tau\right)\left(E_{3}\right)-\left(\nabla_{E_{3}} \tau\right)\left(E_{3}\right)+\tau\left(E_{3}\right) \tau\left(E_{2}\right)+2 \rho_{23} .
\end{gathered}
$$

Therefore

$$
\rho\left(E_{2}, E_{3}\right)=\left[\mathcal{S}(\nabla \tau)-\frac{1}{2} \tau \otimes \tau\right]\left(E_{2}, E_{3}\right) .
$$

If follows from (28) and (29) that

$$
\rho\left(E_{i}, E_{j}\right)=\left[\mathcal{S}(\nabla \tau)-\frac{1}{2} \tau \otimes \tau+\frac{1}{8}\left(2 s+2 \delta \tau+\|\tau\|^{2}\right) g\right]\left(E_{i}, E_{j}\right), \quad i, j=1, \ldots, 4 .
$$

Therefore, for every $X, Y \in T M$,

$$
\rho(X, Y)=\left[\mathcal{S}(\nabla \tau)-\frac{1}{2} \tau \otimes \tau+\frac{1}{8}\left(2 s+2 \delta \tau+\|\tau\|^{2}\right) g\right](X, Y) .
$$

Conversely, suppose that the Ricci tensor of $g$ is given by (30). Then it follows from (28) that $g\left(\mathcal{R}^{D}\left(s_{1}^{+}\right), s_{1}^{-}\right)=0$. An easy computation making use of (6), (8), (44), and (29) gives $g\left(\mathcal{R}^{D}\left(s_{1}^{+}\right), s_{2}^{-}\right)=0$ and $g\left(\mathcal{R}^{D}\left(s_{1}^{+}\right), s_{3}^{-}\right)=0$. Replacing the basis $\left(E_{1}, E_{2}, E_{3}, E_{4}\right)$ by $\left(E_{1}, E_{3}, E_{4}, E_{2}\right)$ and $\left(E_{1}, E_{4}, E_{2}, E_{3}\right)$, we see that $g\left(\mathcal{R}^{D}\left(s_{i}^{+}\right), s_{j}^{-}\right)=0$ for every $i, j=1,2,3$. Therefore if $(M, g)$ is anti-self-dual and its Ricci tensor is given by (30), the almost complex structure $f^{m} \mid \mathcal{Z}_{+} \times \mathcal{Z}_{-}$is integrable.

Remarks 1. In the case when $\tau=0$, i.e., $D$ is the Levi-Civita connection, Theorem 3 coincides with 9 , Theorem 2 (c) and (d)]

2. The identity

$$
\rho(X, Y)=\left[\mathcal{S}(\nabla \tau)-\frac{1}{2} \tau \otimes \tau+\frac{1}{8}\left(2 s+2 \delta \tau+\|\tau\|^{2}\right) g\right](X, Y)
$$

can also be interpreted in terms of the Weyl geometry. Recall that a $n$-dimensional conformal manifold with a Weyl connection $\nabla^{w} w$ is said to be Einstein-Weyl if the symmetric part $R i c^{\text {sym }}$ of the Ricci tensor of $\nabla^{w}$ is proportional to one (hence to every) metric $g$ in the conformal class. The proportionality factor is clearly $s_{g}^{w} / n$, where $s_{g}^{w}$ is the conformal scalar curvature with respect to $g$.

Consider the conformal structure on the Riemannian 4- manifold $M$ determined by the metric $g$, and define a connection $\nabla^{w}$ on $M$ by (18) with $\theta_{g}=-\tau$. Then it follows from (19) and (20) that identity (31) is equivalent to $\nabla^{w}$ being EinsteinWeyl. Hence, if $(M, g, \tau)$ satisfies the conditions of Theorem 3 and $f$ is a smooth function on $M$, then $\left(M, e^{f} g,-d f+\tau\right)$ also satisfies these conditions. 
It has been proved by P. Gauduchon [14] and H. Pedersen - A. Swann 25] that an Einstein-Weyl structure on a compact self-dual manifold is closed. Thus, we have the following.

Corollary 3. If the base manifold $M$ is compact and the almost complex structure $g^{m}$ on $\mathcal{Z}_{+} \times \mathcal{Z}_{-}$(resp. $\left.\mathcal{Z}_{-} \times \mathcal{Z}_{+}\right), m=1$ or 2 , is integrable, then $d \tau=0$.

Examples. 1. Combining the above mentioned result by P. Gauduchon and H. Pedersen - A. Swann with results due to H. Pedersen - A. Swan in [26, D. Calderbank and H. Pedersen have given the following classification of compact self-dual Einstein-Weyl manifold [5, Theorem 9.8]: A compact self-dual Einstein-Weyl 4manifold is isometric to $S^{4}, \mathbb{C P}^{2}$, or an Einstein manifold of negative scalar curvature, or is covered by a flat torus, a $K 3$-surface or a coordinate quaternionic Hopf surface. Thus, these manifolds satisfy the conditions of Theorem 3 , We refer to [5] and the literature therein for details.

2. Consider the Lie group $G_{\lambda}$ with the (anti-) self-dual metric $g_{k}, k=1,2$, defined above. Then a left-invariant 1-form $\tau$ satisfies identity (31) if and only if $\tau=0$ and $k=2$ (i.e., the metric $g_{2}$ is Einstein).

3. Other non-compact examples of Einstein-Weyl manifolds can be found in [4] (see also [5]).

\section{Acknowledgements}

The author would like to thank V. Apostolov and A. Swan for helpful discussions and suggestions. He is also grateful to the referee whose remarks helped to improve the final version of the paper.

\section{REFERENCES}

[1] M. F. Atiyah, N. J. Hitchin, I. M. Singer, Self-duality in four-dimensional Riemannian geometry, Proc. Roy. Soc. London, Ser.A 362 (1978), 425-461.

[2] L. Bérard-Bergery, T. Ochiai, On some generalization of the construction of twistor spaces, in Global Riemannian geometry (T.J.Willmore, N.J.Hitchin, eds.), an L.M.S. symposium held in Durham, July 1983, Ellis Horwood, 1984, pp.52-59

[3] A. Besse, Einstein manifolds, Classics in Mathematics, Springer-Verlag, 2008.

[4] G. Bonneau, Einstein-Weyl structures corresponding to diagonal Kähler Bianchi IX metrics, Class. Quantum Grav. 14 (1997), 2123-2135.

[5] D.M.J. Calderbank, H. Pedersen, Einsten-Weyl geometry, Surveys in differential geometry: essays on Einstein manifolds, pp. 387-423, Surv. Differ. Geom., 6, Int. Press, Boston, MA, 1999.

[6] J. Davidov, O. Muskarov, Existence of holomorphic functions on twistor spaces, Bull. Soc. Math. Belgique 40, fasc. I-ser.B (1989), 131-151.

[7] J. Davidov, Harmonic almost Hermitian structures, in Special metrics and group actions in geometry (S.Chiossi, A.Fino, F.Podestà, E.Musso, L.Vezzoni, eds.), Proceedings of the workshop "New perspectives in differential geometry: special metrics and quaternionic geometry", held in Rome, 16-20 November, 2015, Springer INdAM Series 23, SpringerVerlag, 2017, pp. 129-159.

[8] J. Davidov, Generalized metrics and generalized twistor spaces, Math. Z. 291 (2019), 17-46.

[9] G. Deschamps, Espace de twisteurs des structures complexes généralisées, Math. Z. 279 (2015), 703-721.

[10] P. Dubois-Violette, Structures complexes au-dessus des variétés, applications, in Mathématique et physique, Séminaire de l'Ecole Normale Superieur, Paris 1979-1982 (L.Boutet de Monvel, A.Douady, J.-L.Verdier, eds.), Progress in Math., vol. 37, Birkhäuser, Boston Basel Stuttgart, 1983, pp. 1-42.

[11] J. Eells, S. Salamon, Twistorial constructions of harmonic maps of surfaces into fourmanifolds, Ann. Scuola Norm. Sup. Pisa, ser.IV, 12 (1985), 589-640. 
[12] A. C. Ferreira, Einstein four-manifolds with skew torsion, J. Geom. Phys. 61 (2011), 23412351.

[13] P. Gauduchon, Structures de Weyl et théorèms d'annualation sur une variété conforme autoduale, Ann. Scuola Norm. Sup., ser.IV, 18 (1991), 563-629.

[14] P. Gauduchon, Structures de Weyl-Einstein, espaces de twisteurs at variétés de type $S^{1} \times$ $S^{3}$, J. reine angew. Math. 469 (1995), 1-50.

[15] M. Gualtieri, Generalized complex geometry, Ph.D. thesis, St John's College, University of Oxford, 2003, arXiv: math.DG/0401221

[16] N. Hitchin, Generalized Calabi-Yau manifolds, Quart. J. Math. 54 (2004), 281-308.

[17] N. Hitchin, Generalized geometry - an introduction, in Handbook of pseudo-Riemannian geometry and suppersymmetry (V. Cortes, ed.), IRMA Lectures in Mathematics and Theoretical Physics 16, European Mathematical Society, Zürich, 2010, pp. 185-208.

[18] I. Kim, Almost Kähler anti-self-dual metrics, Ph.D. thesis, Stony Brook University, May 2014, available at www.math.stonybrook.edu/alumni/2014-Inyong-Kim.pdf; see also arXiv: math.DG/1511.07656.

[19] J. Kim, M. Pontecrovo, A new method of constructiong scalar-flat Kähler surfaces, J. Diff. Geom. 41 (1995), 440-477.

[20] T. Koda, Self-dual and anti-self-dual Hermitian surfaces, Kodai Math. J. 10 (1987), 335342.

[21] C. LeBrun, Poon's self-daul metrics and Kähler geometry, J. Diff. Geom. 28 (1998), 341343.

[22] C. LeBrun, Anti-self-dual Hermitian metrics on blow-up Hopf surfaces, Math. Ann. 289 (1991), 383-392.

[23] C. LeBrun, Anti-self-dual metrics and Kähler geometry, Proceedings of the International Congress of Mathematics, Vol. 1,2 (Zürich, 1994), pp. 498-507, Birkhäuser, Basel, 1995.

[24] N. O'Brian, J. Rawnsley, Twistor spaces, Ann. Glob. Anal. Geom. 3 (1985), 29-58.

[25] H. Pedersen, A. Swann, Riemannian submersions, four manifolds and Einstein-Weyl geometry, Proc. London Math. Soc. 66 (1993), 381-399.

[26] H. Pedersen, A. Swann, Einsten-Weyl geometry, the Bach tensor and conformal scalar curvature, J. reine andew. Math. 441 (1993), 99-113.

[27] Y. Rollin, M. Singer, Non-minimal scalar-flat Kähler surfaces and parabolic stability, Invent. Math. 162 (2005), 235-270.

[28] I. M. Singer, J. A. Thorpe, The curvature of 4-dimensional Einstein spaces, in Papers in Honor of K. Kodaira, Princeton University Press (Princeton), 1969, pp. 355-365.

[29] V. de Smewdt, S. Salamon, Anti-self-dual Lie groups, Differential geometry and integrable systems (Tokyo, 2000), pp. 63-75, Contemp. Math. 308, Amer. Math. Soc. Providence, RI, 2002.

[30] I.Vaisman, Some curvature properties of complex surfaces, Ann.Mat.Pura Appl. 32 (1982), $1-18$.

[31] F. Witt, Special metric structures and closed forms, Ph.D. thesis, Oxvord University, 2004, arXiv: math.DG/0502443

Institute of Mathematics and Informatics, Bulgarian Academy of Sciences, Acad. G.Bonchev Str. Bl.8, 1113 Sofia, Bulgaria

E-mail address: jtd@math.bas.bg 